\title{
Denture-Associated Oral Microbiome and Periodontal Disease Causing an Anaerobic Pyogenic Liver Abscess in an Immunocompetent Patient: A Case report and Review of the Literature
}

\author{
Muhammad Bader Hammami ${ }^{\mathrm{a}, \mathrm{f}}$, Elizabeth M. Noonan ${ }^{\mathrm{b}}$, Anuj Chhaparia ${ }^{\mathrm{c}}$, \\ Feras Al Khatib ${ }^{\mathrm{d}}$, Juri Bassuner ${ }^{\mathrm{e}}$, Christine Hachem ${ }^{\mathrm{a}}$
}

\begin{abstract}
Pyogenic liver abscesses (PLA) develop from the spread of infection through the portal circulation, biliary infections or arterial hematogenous seeding in the setting of systemic infections. PLA are often poly-microbial and are uncommonly reported to be due to anaerobic species. We report the case of a previously healthy, immunocompetent 63-year-old man with hepatic abscesses as a result of Fusobacterium nucleatum periodontal disease. In addition, a systemic review of the literature is performed. Fusobacterium is a very rare cause of PLA in immunocompetent hosts with only a handful of cases reported in the literature. Although anaerobic infections such as Fusobacterium most often occur in immunocompromised individuals, clinicians should have a high index of suspicion in immunocompetent patients with periodontal disease or chronic stomatitis.
\end{abstract}

Keywords: Pyogenic; Anaerobe; Liver abscess; Fusobacterium nucleatum; Denture; Periodontitis

\section{Introduction}

Pyogenic liver abscesses (PLA) most commonly develop fol-

Manuscript submitted March 17, 2018, accepted May 3, 2018

${ }^{a}$ Department of Internal Medicine, Division of Gastroenterology and Hepatology, Saint Louis University School of Medicine, St Louis, MO, USA

${ }^{b}$ Department of Pediatrics, Saint Louis University School of Medicine, Saint Louis, MO, USA

'Department of Internal Medicine, Saint Louis University School of Medicine, Saint Louis, MO, USA

${ }^{\mathrm{d}}$ Southern Illinois University, School of Dental Medicine, Alton, IL, USA

eDepartment of Radiology, Saint Louis University School of Medicine, Saint Louis, MO, USA

${ }^{\mathrm{f}}$ Corresponding Author: Muhammad B. Hammami, Division of Gastroenterology and Hepatology, Saint Louis University School of Medicine, 1402 S. Grand Blvd., St Louis, MO 63104, USA.

Email: Muhammad.Hammami@Health.SLU.Edu

doi: https://doi.org/10.14740/gr1006w lowing peritonitis due to intra-abdominal bowel leakage with subsequent spread to the liver through the portal circulation [1] or via direct spread from biliary infections $[2,3]$. They may also result from arterial hematogenous seeding in the setting of systemic infections, such as in cases of endocarditis or septic thrombophlebitis [4]. PLA are often poly-microbial and are uncommonly reported to be due to anaerobic species $[5,6]$. We report the case of an otherwise healthy immunocompetent patient who developed multiple hepatic abscesses caused by Fusobacterium nucleatum. Additionally, we review and summarize the literature on PLA.

\section{Case Report}

A 63-year-old healthy African-American male presented with a 4-day history of food aversion, subjective fevers, chills, chest and abdominal pain. His past medical history was significant for remote history of tobacco and cocaine use and was negative for diabetes mellitus or other medical illnesses. He was not on any prescription or over-the-counter medications. On presentation, he was found to have a fever of $38.3^{\circ} \mathrm{C}$, but he was hemodynamically stable. A detailed physical exam was noted for poor dentition but otherwise was unremarkable. Laboratory testing revealed a white cell count of $22.4 \times 10^{9} / \mathrm{L}$, aspartate aminotransferase of 93 units/L, alanine aminotransferase of 110 units/L and total bilirubin of $1.2 \mathrm{mg} / \mathrm{dL}$. Contrasted computed tomography of the chest, abdomen and pelvis revealed multiple indeterminate hypo-attenuating lesions throughout the liver (Fig. 1). Further imaging with an abdominal sonogram identified multiple complex hypoechoic foci throughout the liver. Magnetic resonance imaging of the abdomen demonstrated multiple cystic lesions with enhancing internal septations and marked diffusion restriction. The largest lesion measured $4.5 \mathrm{~cm} \times 4.1 \mathrm{~cm} \times 3.8 \mathrm{~cm}$ (Fig. 2).

Empiric treatment with broad-spectrum antibiotics was initiated on hospitalization day 1 and resulted in immediate resolution of his fever. Successful aspiration from one of the abscess cavities and placement of three drainage catheters in the right hepatic lobe was achieved by interventional radiology. Drainage from the hepatic abscess grew Fusobacterium nucleatum, and antibiotics were narrowed based on culture 


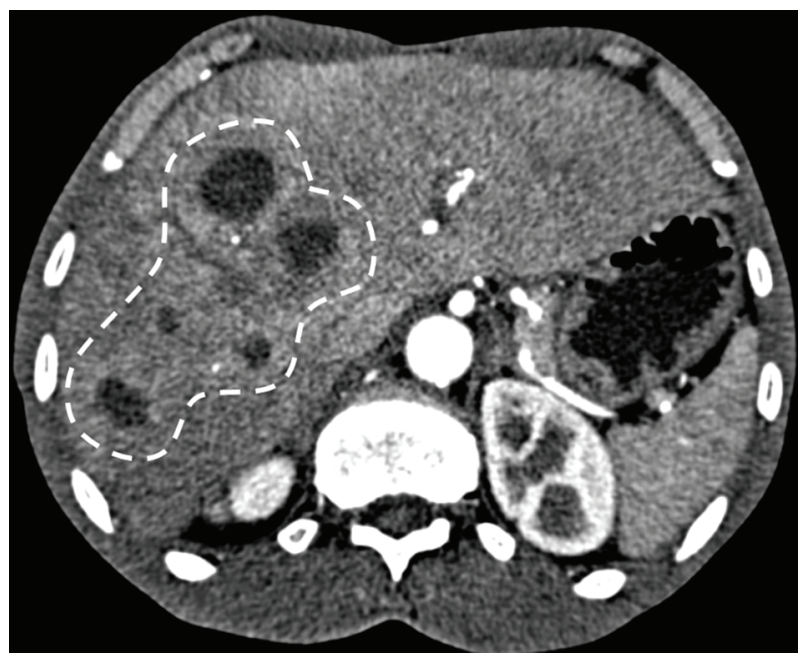

Figure 1. CT with contrast showing multiple hypoattenuating lesions in the liver (dashed line).

and sensitivities. The liver aspirate testing for acid-fast organisms, aerobic and fungal cultures was negative. Stool cultures and examination for ova, parasites, giardia, cryptosporidium, clostridium difficile and entamoeba were all negative. Further infectious disease evaluation was unremarkable, including negative blood and urine cultures, and testing for viral hepatitis and human immunodeficiency virus was negative. Furthermore, tumor markers for alpha-fetoprotein, cancer antigen 19-9 and carcinoembryonic antigen were unremarkable. A trans-thoracic echocardiogram revealed no evidence of valvular vegetations to suggest endocarditis. Furthermore, endoscopic evaluation by colonoscopy revealed two $4-8 \mathrm{~mm}$ adenomatous polyps in the rectum and mild diverticulosis in the sigmoid colon but without evidence of prior or current inflammation or infection. Finally, a panorex demonstrated multiple missing teeth and lucency around the root of a left mandibular premolar, likely representing a periapical abscess (Fig. 3).

Output from the hepatic drains continued to decrease, and the drains were eventually removed on hospitalization day 14 . Affected teeth were extracted prior to discharge, and antibiotics were transitioned to an oral route. He was discharged home after a 4-week hospitalization. At 6 weeks post-hospitalization follow-up, he remained asymptotic and liver enzymes trended down to normal levels. Clinically significant labs with changes are shown in Table 1. Repeat cross-sectional imaging demonstrated a significant interval decrease in rim enhancement and size of the known liver abscesses, compatible with treatment response (Fig. 4).

\section{Discussion}

PLA account for nearly $50 \%$ of visceral abscess cases in the USA [7]. To date, the largest population-based study on PLA in the USA estimated the annual incidence of PLA at 3.6 cases per 100,000 population, with predominance among men and the elderly [5]. However, there is significant geographic variation globally, as demonstrated by the much higher incidence

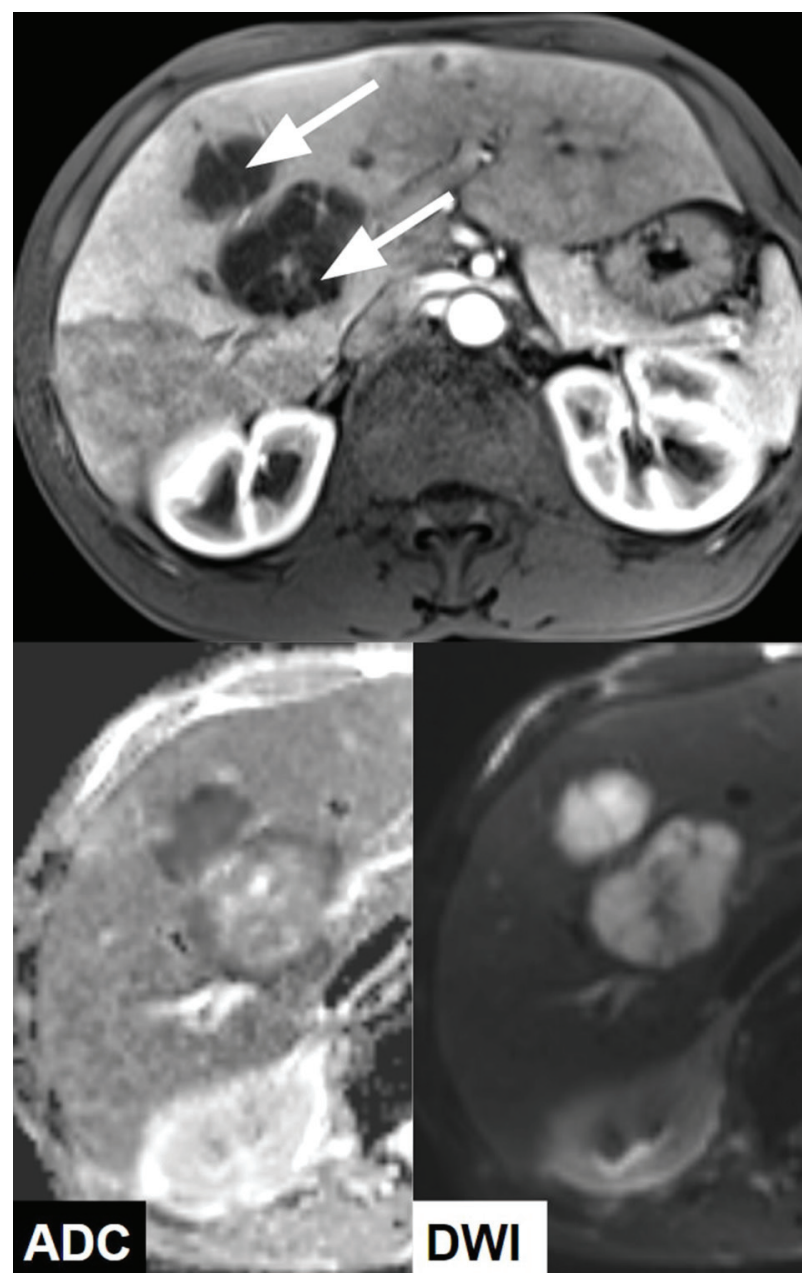

Figure 2. MRI with contrast demonstrating multiple hepatic cystic lesions with enhancing septa (arrows) and marked diffusion restriction (lesions are hypointense on apparent diffusion coefficient (ADC) and hyperintense on diffusion-weighted imaging (DWI) compatible with abscess).

reported in Taiwan of up to 17.6 per 100,000 individuals [8]. In addition, there is also variation among different disease groups. According to research published by Clinical Infectious Disease (CID) in 2007, individuals with diabetes are at 3.6 times greater risk for PLA [9]. In a retrospective review completed by CID in 2004, out of 79 cases of PLA, $43 \%$ of patients had underlying biliary disease [1].

The most common clinical presentation of PLA includes fever, chills, malaise and right upper abdominal pain. The patient in our case presented with shortness of breath and chest pain, which may be attributed to diaphragmatic irritation from liver enlargement. Physical examination findings often include right upper quadrant abdominal tenderness, jaundice and hepatomegaly. In our case, the patient's physical exam was relatively benign with exception of fever and poor dentition. With PLA, common laboratory abnormalities include (in order of prevalence): hypoalbuminemia, elevated $\gamma$-glutamyltransferase, elevated alkaline phosphatase level and leukocytosis $[6,10]$, all of which were observed in our case (Table 2). Approximately half of patients will have elevated aspartate and alanine ami- 


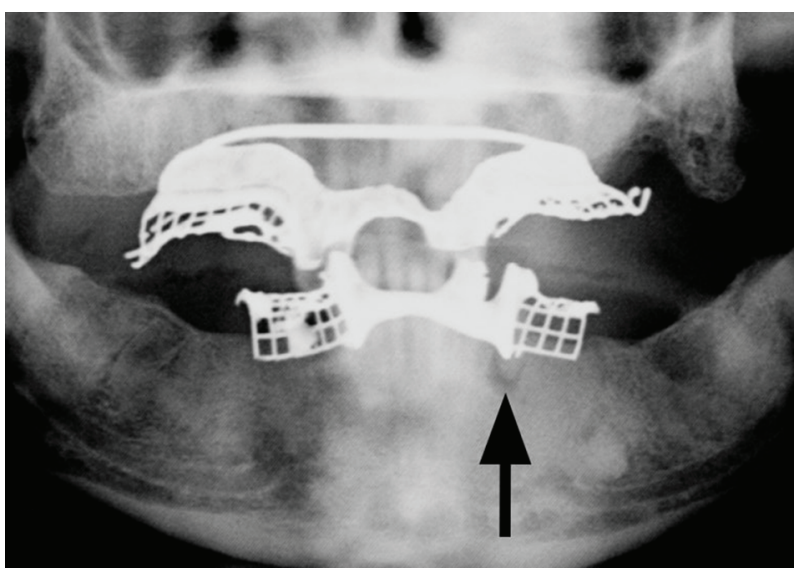

Figure 3. Panorex demonstrating multiple missing teeth with lucency around the root of a left mandibular premolar (arrow), likely representing a periapical abscess.

notransferases, elevated total bilirubin levels, normocytic anemia and hyponatremia [6]. Blood cultures are only positive in $30-60 \%$ of cases, but organisms are isolated from the majority $(70-80 \%)$ of abscess aspirates $[6,11]$.

Meddings et al conducted a study reviewing nearly 18,000 cases of PLA in the USA and found that the most commonly recorded bacterial infections were due to Streptococcus species (29.5\%) and Escherichia coli (18.1\%) [5]. In contrast, anaerobic species were uncommon $(8.6 \%)$, but were likely underreported because of their difficulty to culture. Kaplan et al conducted a population-based surveillance study in the Calgary Health Region and determined that $49 \%$ of PLA infections were polymicrobial. The most commonly identified organisms were Streptococcus milleri group (44\%), Klebsiella species $(27 \%)$ and anaerobes $(20 \%)$. The anaerobes included Clostridium perfringens (4\%), Bacteroides species (4\%), Peptostreptococcus species (3\%), Fusobacterium necrophorum (3\%), Actinomyces species (1\%), Prevotella species (1\%), Lactobacillus (1\%) and Veillonella (1\%) [7]. Amebic abscesses account for the minority of hepatic abscesses in the United States but should be considered in patients with recent travel to an endemic area. Interestingly, in Asian populations, Klebsiella pneumonia has been identified as the most common cause of PLA $[1,12]$.

Percutaneous drainage, along with targeted antimicrobial therapy, is the mainstay of treatment. Implications for surgi-

Table 1. Laboratory Data: Hospitalization Days 1, 28 and 64

\begin{tabular}{llll}
\hline Test (normal range) & Day 1 & Day 28 & Day 64 \\
\hline WBC $\left(3.5 \times 10^{3}-10.5 \times 10^{3} / \mu \mathrm{L}\right)$ & 28.5 & 7.6 & 5.4 \\
Albumin $(3.4-5 \mathrm{~g} / \mathrm{dL})$ & 2.7 & 2.9 & 3.2 \\
ALT $(0-55$ units/L) & 117 & 29 & 17 \\
AST $(5-34$ units/L) & 88 & 28 & 20 \\
ALP $(40-150$ units/L) & 179 & 182 & 87 \\
Total bilirubin $(0.2-1.2 \mathrm{mg} / \mathrm{dL})$ & 1.6 & 0.4 & 0.4 \\
\hline
\end{tabular}

WBC, white blood cell; ALT, alanine aminotransferase; AST, aspartate aminotransferase; ALP, alkaline phosphatase.

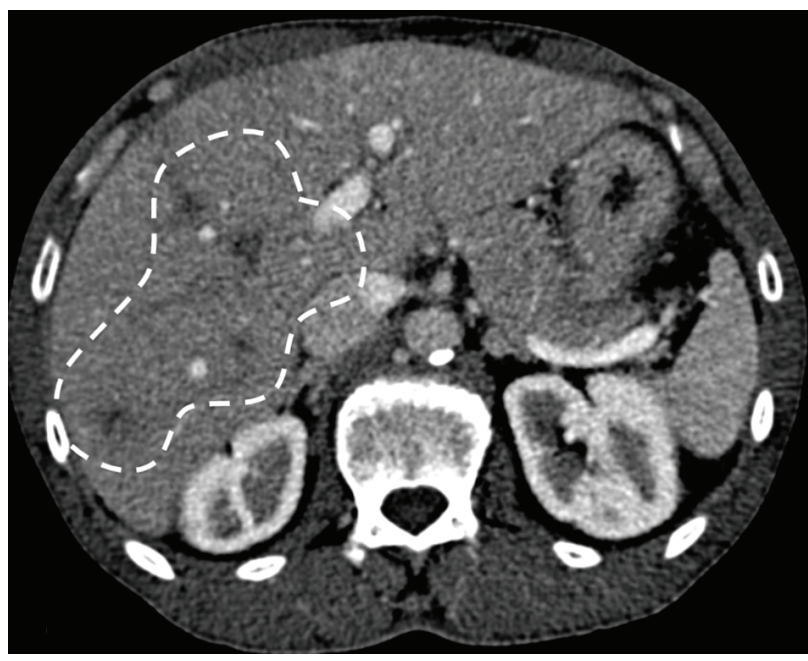

Figure 4. CT with contrast showing interval decrease in rim enhancing fluid collections at 10 weeks (dashed line).

cal drainage include abscess rupture, incomplete percutaneous drainage, multi-loculated abscesses and inadequate clinical response 4 - 7 days after percutaneous drainage. Recommended antibiotic treatment includes parenteral therapy for $2-3$ weeks followed by oral therapy for $2-6$ weeks or until clinical, laboratory and radiographic studies show resolution of the abscess [13]. The overall mortality rate associated with PLA has improved significantly since 1980 , from greater than $50 \%$ to $10 \%$ in developed countries. Independent risk factors for mortality include the need for open surgical drainage, the presence of anaerobic infection, bacteremia or septicemia, and comorbidities such as advanced age, cirrhosis, chronic renal failure and malignancy [5].

The periodontium, also called alveolodental membrane or peridental membrane, is the tissue that surrounds and supports the teeth in the maxillary and mandibular bones, including the gingiva, cementum, periodontal ligament, and alveolar and supporting bone [14]. Periodontitis is defined as inflammation of the periodontium caused by bacteria that infect the roots of the teeth and surrounding gum crevices, producing bleeding, pus formation, and gradual loss of bone and tissue that support the teeth [15]. Dentures are an artificial replacement of one or several of the teeth (partial denture) or all of the teeth (full denture) of either or both jaws [16]. Recent developments in the field of microbiology have isolated the main microorganisms involved in the etiology of periodontitis. These are mainly Gram-negative species including: Aggregatibacter actinomycetemcomitans, Tannerella forsythia, Porphyromonas gingivalis, Prevotella intermedia, Campylobacter rectus, Treponema denticola and Fusobacterium nucleatum. Virulence factors produced by these microorganisms have been identified, and their role in periodontal destruction is well established [17].

Fusobacterium is an anaerobic, Gram-negative, invasive, adherent, non-motile, non-spore forming, pro-inflammatory, spindle-shaped rod. Fusobacterium nucleatum and Fusobacterium necrophorum are the most common species of Fusobacterium responsible for human infection. Fusobacterium nucleatum is indigenous to the human oral flora and plays a 


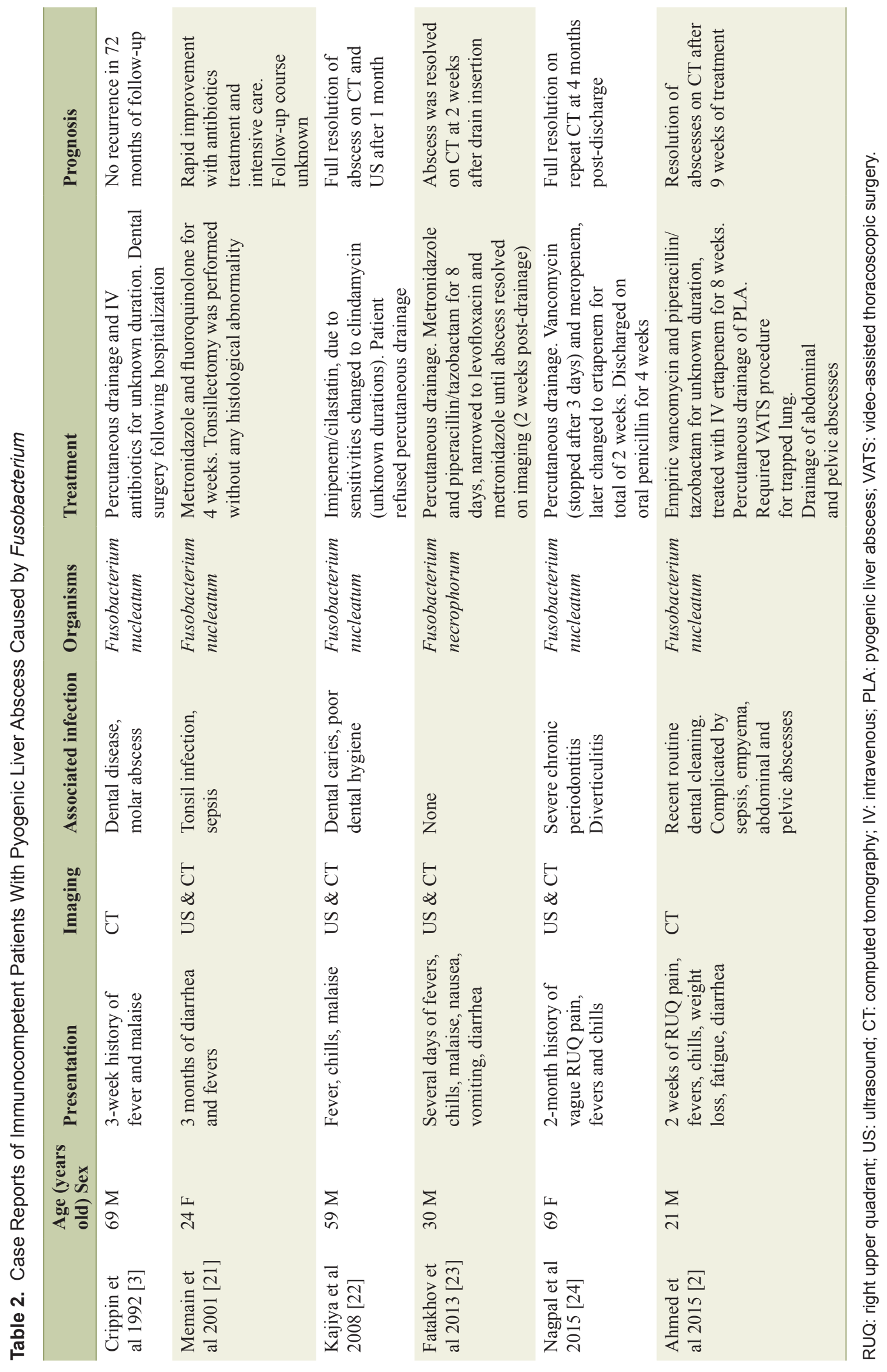


major role in periodontal disease [18]. This anaerobe has also been associated with many other infections, including peritonsillar abscesses and appendicitis [2]. Interestingly, a study conducted by Genome Research in 2012 showed a highly significant prevalence of Fusobacterium nucleatum in human colorectal tumor specimens [19]. However, only six cases of mono-microbial liver abscesses have been reported to be due to Fusobacterium in immunocompetent hosts (Table 2). These cases were published between 1977 and 2015. Out of the six cases, five were linked to periodontal disease; in one of the cases, a source of the infection was never discovered. In their research, Shi et al also found a significant increase in the bacterial load of Fusobacterium nucleatum in patients who wear dentures, especially those who develop stomatitis [20]. In our patient, given the right hepatic lobe distribution, bacteremic spread of infection originating from a dental source was likely, given the identified organism and the patient's history of tooth abscess.

\section{Conclusions}

Fusobacterium is a rare cause of PLA in immunocompetent individuals and is commonly associated with periodontitis, as observed in this case. As demonstrated by Table 2, other case reports have also shown a correlation between anaerobic PLA with Fusobacterium and dental disease in otherwise healthy individuals. Our patient likely acquired this invasive strain of Fusobacterium from his dental abscess which was identified and treated during his hospitalization, in the setting of chronic denture use. Thus, chronic denture use should be considered as a possible contributing factor for infection in similar cases, and may have been overlooked in previous case reports.

\section{Conflict of Interest}

No potential conflicts of interest.

\section{Financial Support}

No financial support.

\section{References}

1. Rahimian J, Wilson T, Oram V, Holzman RS. Pyogenic liver abscess: recent trends in etiology and mortality. Clin Infect Dis. 2004;39(11):1654-1659.

2. Ahmed Z, Bansal SK, Dhillon S. Pyogenic liver abscess caused by Fusobacterium in a 21-year-old immunocompetent male. World J Gastroenterol. 2015;21(12):37313735 .

3. Crippin JS, Wang KK. An unrecognized etiology for pyogenic hepatic abscesses in normal hosts: dental disease. Am J Gastroenterol. 1992;87(12):1740-1743.

4. Wong WM, Wong BC, Hui CK, Ng M, Lai KC, Tso WK,
Lam SK, et al. Pyogenic liver abscess: retrospective analysis of 80 cases over a 10-year period. J Gastroenterol Hepatol. 2002;17(9):1001-1007.

5. Meddings L, Myers RP, Hubbard J, Shaheen AA, Laupland KB, Dixon E, Coffin C, et al. A population-based study of pyogenic liver abscesses in the United States: incidence, mortality, and temporal trends. Am J Gastroenterol. 2010;105(1):117-124.

6. Kaplan GG, Gregson DB, Laupland KB. Populationbased study of the epidemiology of and the risk factors for pyogenic liver abscess. Clin Gastroenterol Hepatol. 2004;2(11):1032-1038.

7. Altemeier WA, Culbertson WR, Fullen WD, Shook CD. Intra-abdominal abscesses. Am J Surg. 1973;125(1):7079.

8. Tsai FC, Huang YT, Chang LY, Wang JT. Pyogenic liver abscess as endemic disease, Taiwan. Emerg Infect Dis. 2008;14(10):1592-1600.

9. Thomsen RW, Jepsen P, Sorensen HT. Diabetes mellitus and pyogenic liver abscess: risk and prognosis. Clin Infect Dis. 2007;44(9):1194-1201.

10. Santos-Rosa OM, Lunardelli HS, Ribeiro-Junior MA. Pyogenic Liver Abscess: Diagnostic and Therapeutic Management. Arq Bras Cir Dig. 2016;29(3):194-197.

11. Huang CJ, Pitt HA, Lipsett PA, Osterman FA, Jr., Lillemoe KD, Cameron JL, Zuidema GD. Pyogenic hepatic abscess. Changing trends over 42 years. Ann Surg. 1996;223(5):600-607; discussion 607-609.

12. Liu L, Chen W, Lu X, Zhang K, Zhu C. Pyogenic liver abscess: a retrospective study of 105 cases in an emergency department from East China. J Emerg Med. 2017;52(4):409-416.

13. Longworth S, Han J. Pyogenic liver abscess. Clinical Liver Disease. 2005;6(2):51-54.

14. Andrei M, Dinischiotu A, Didilescu AC, Ionita D, Demetrescu I. Periodontal materials and cell biology for guided tissue and bone regeneration. Ann Anat. 2018;216:164169.

15. Savage A, Eaton KA, Moles DR, Needleman I. A systematic review of definitions of periodontitis and methods that have been used to identify this disease. J Clin Periodontol. 2009;36(6):458-467.

16. Milward P, Katechia D, Morgan MZ. Knowledge of removable partial denture wearers on denture hygiene. $\mathrm{Br}$ Dent J. 2013;215(10):E20.

17. Pokrowiecki R, Mielczarek A, Zareba T, Tyski S. Oral microbiome and peri-implant diseases: where are we now? Ther Clin Risk Manag. 2017;13:1529-1542.

18. Kapatral V, Anderson I, Ivanova N, Reznik G, Los T, Lykidis A, Bhattacharyya A, et al. Genome sequence and analysis of the oral bacterium Fusobacterium nucleatum strain ATCC 25586. J Bacteriol. 2002;184(7):2005-2018.

19. Castellarin M, Warren RL, Freeman JD, Dreolini L, Krzywinski M, Strauss J, Barnes R, et al. Fusobacterium nucleatum infection is prevalent in human colorectal carcinoma. Genome Res. 2012;22(2):299-306.

20. Shi B, Wu T, McLean J, Edlund A, Young Y, He X, Lv H, et al. The Denture-Associated Oral Microbiome in Health and Stomatitis. mSphere. 2016;1(6). 
21. Memain N, Arvaniti K, Bruneel F, Leport C, Wolff M, Regnier B. [Septic shock with liver abscess in an immunocompetence patient. Presentation of an unusual Fusobacterium nucleatum infection]. Presse Med. 2001;30(36):1777-1779.

22. Kajiya T, Uemura T, Kajiya M, Kaname H, Hirano R, Uemura N, Tei C. Pyogenic liver abscess related to dental disease in an immunocompetent host. Intern Med.
2008;47(7):675-678

23. Fatakhov E, Patel MK, Santha S, Koch CF, Jr. Fusobacterium necrophorum: a rare cause of hepatic abscess in an immunocompetent individual. BMJ Case Rep. $2013 ; 2013$.

24. Nagpal SJ, Mukhija D, Patel P. Fusobacterium nucleatum: a rare cause of pyogenic liver abscess. Springerplus. 2015;4:283. 